\title{
苯偶姻单酯无溶剂微波加热合成 2-取代-4,5-二芳基咪唑衍生物
}

\author{
李宝志顾强赵天琦贺元辉佟亚楠张玉敏* \\ (吉林大学化学学院 长春 130012)
}

\begin{abstract}
摘要 以醛为原料, 在维生素 $\mathrm{B}_{1}$ 作用下，经安息香缩合反应制得相应的苯偶姻衍生物，进而以此为原料与不同的酰化 试剂酯化生成苯偶姻单酯. 苯偶姻单酯与醋酸铵附着在固载体酸性氧化铝上, 在无溶剂条件下微波加热合成了 17 种 2取代-4,5-二芳基咪唑，其中 7 种未见文献报道. 该方法具有反应条件温和、反应时间短，且无需有机溶剂，是一种节能 环保、易操作的合成方法. 另外, 所合成化合物的结构通过 IR、高分辨质谱和核磁共振谱进行了确认.

关键词 2-取代-4,5-二芳基咪唑; 苯偶姻单酯; 微波合成; 固体支持; 无溶剂; 酯化
\end{abstract}

\section{Microwave-Assisted Synthesis of 2-Substituted-4,5-diarylimidazoles from Benzoin Carboxylate Derivatives under Solvent-Free Conditions}

\author{
Li, Baozhi Gu, Qiang Zhao, Tianqi He, Yuanhui Tong, Yanan Zhang, Yumin* \\ (College of Chemistry, Jilin University, Changchun 130012)
}

\begin{abstract}
Benzoin carboxylate derivatives were synthesized with the aromatic aldehydes as raw material in the presence of the catalyst $\mathrm{VB}_{1}$. And then, benzoin carboxylate derivatives reacted with different acylating reagents under mild reaction conditions, and benzoin carboxylates were obtained. Further, 2-substituted-4,5-di(aryl)imidazoles were produced from benzoin carboxylates in the presence of solid supports $\mathrm{Al}_{2} \mathrm{O}_{3}$ under solvent-free and microware assisted condition, and seven of them have not been reported. The reaction possessed many advantages such as mild reaction conditions, short reaction time, and no organic solvents consumed. Therefore, it is a facile and efficient synthetic method. Moreover, the synthesized compounds were characterized by ${ }^{1} \mathrm{H}$ NMR, ${ }^{13} \mathrm{C}$ NMR, IR, HRMS techniques.
\end{abstract}

Keywords 2-substituted-4,5-di(aryl)imidazoles; benzoin carboxylates; microwave-assisted synthesis; solid-support; solvent-free; esterification

咪唑是重要的有机杂环化合物之一, 它含有两个氮 原子，属于芳香性化合物. 因其含有仲胺结构，所以咪 唑具有两性, 即弱酸弱碱性. 另外, 咪唑是富 $\pi$ 电子的 杂环, 能传递电子和质子, 化学性质活泼, 是许多酶的 活性中心, 具有优良的药学和与金属离子配位的性能. 因此，咪唑及其衍生物在医药、农药、材料、金属离子 检测和有机合成等方面用途广泛. 它可以直接参与某些 生物化学反应合成杀菌剂、抑制剂、除草剂、植物增长 剂、药物缓蚀剂等 ${ }^{[1]}$. 在离子检测中, 可以用来分析检测 无机金属离子和医药中有机化合物的含量 ${ }^{[2]}$. 另外, 咪 唑环可以发生分子内电荷转移, 具有很好的电子传输性 能和热稳定性, 因此咪唑类化合物及其衍生物在光致变 色、激光染料、电致发光等发光功能材料中备受关注 ${ }^{[3,4]}$. 可以做成优良的有机非线性光学材料 $[5,6]$ 、质子传输材 料 ${ }^{[7,8]}$ 和发光材料 ${ }^{[9]}$ 等.
咪唑及其衍生物的合成方法有很多 ${ }^{[10]}$. 如德布斯 法, 魏登一哈根法, 克莱森重排法, 瓦拉赫法, 咪唑啉氧 化法, 沃尔一马克瓦尔德法和狄尔斯-阿尔德尔法等. 这 些方法操作比较繁琐, 反应必须在溶剂和催化剂的存在 下进行, 反应时间较长, 且产率较低. 最近, 研究者基 于咪唑衍生物的广泛用途, 研究出一些合成咪唑的新方 法 $^{[11]}$, 如以离子液体、磷酸二氢钠、双乙酸基碘代苯 (diacetoxy iodobenzene)、固体酸等为催化剂的传统合成 方法和在催化剂存在下的微波辅助合成法等, 这些合成 方法都是以二苯基乙二酮和醛，或者安息香和醛为原 料, 在相应的催化剂和溶剂作用下合成目标产物. 另据 文献[12，13]报道，氧化铝和二氧化硅对反应物和微波 具有很好的分散性, 并且最近几年微波反应在有机合成 中的应用备受关注 ${ }^{[12 \sim 14]}$. 因此本文采用氧化铝作为反 应物和微波分散剂, 以苯偶姻单酯及取代苯偶姻单酯为

*E-mail: zhang_ym@jlu.edu.cn

Received January 10, 2012; revised March 10, 2012; published online April 24, 2012. 
原料，在微波辅助的无溶剂条件下成功地合成了 17 种 三取代咪唑衍生物. 其合成路线如 Scheme 1 所示.

该方法反应时间短，不使用溶剂，操作简单，因此 本方法具有节能、环保等优点. 同时利用红外光谱、高 分辨率质谱、核磁共振氢谱、碳谱对所合成的化合物进 行了表征.

\section{1 实验部分}

\section{1 仪器与试剂}

熔点采用北京泰克 XT-4 熔点仪测定(温度计未校 正); 氢谱和碳谱用 Bruker500 型或 Mercury-300 型核磁 共振波谱仪测定, TMS 为内标, $\mathrm{CDCl}_{3}$ 或 DMSO- $d_{6}$ 为溶 剂; 高分辨质谱利用 Agilent1290-micrOTOF Q II 高分辨 液质联用分析仪进行测定; 红外光谱由日本岛津的 Shimadzu IRAffinity-1 型红外光谱仪测定, 采用 KBr 压 片法; 微波反应用四川大学研制的 MCL-3 型微波反应
仪. 本实验所用试剂均为分析纯试剂. 取代苯偶姻单酯 衍生物的合成参见文献 [15]; 酰氯的合成参见文献 [16, 17].

\section{2 苯偶姻单酯 $3 a \sim 3 q$ 的合成}

在装有温度计, 电磁搅拌的 $100 \mathrm{~mL}$ 三颈瓶中, 加

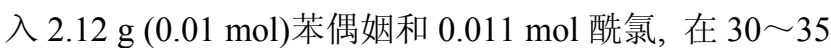
${ }^{\circ} \mathrm{C}$ 的水浴条件下, 搅拌 $5 \mathrm{~min}$ 后缓慢滴加吡定, 滴完后, 再保温反应 $1 \mathrm{~h}$. 趁热将反应混合液到入约 $200 \mathrm{~mL}$ 蒸馏 水中，搅拌片刻，抽滤，产物依次用乙醇 $(5 \mathrm{~mL} \times 2)$ 、冰 水 $(5 \mathrm{~mL} \times 3)$ 洗涤，得到产物粗品，然后用 $95 \%$ 的乙醇水 溶液重结晶, 真空干燥, 计算收率.

\subsection{2-取代-4,5-二芳基咪唑(4a 4q)的合成}

称取 $1 \mathrm{mmol}$ 苯偶姻单酯或取代苯偶姻单酯、3 $\mathrm{mmol}$ 醋酸铵和 $1 \mathrm{~g}$ 三氧化二铝放入研钵中, 经充分研磨 混均后, 将其加入到 $100 \mathrm{~mL}$ 圆底烧瓶中, 在微波中加
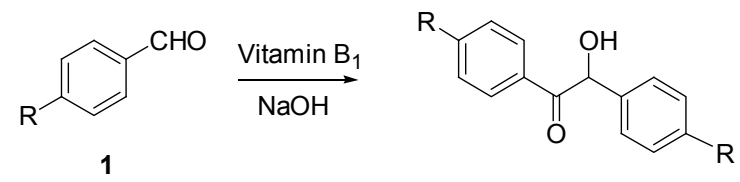<smiles>[R]C(=O)OC(C(=O)c1ccc([R])cc1)c1ccc([R])cc1</smiles>

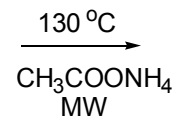

$3 a \sim 3 q$

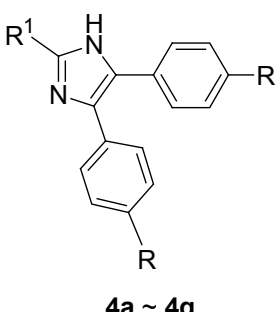

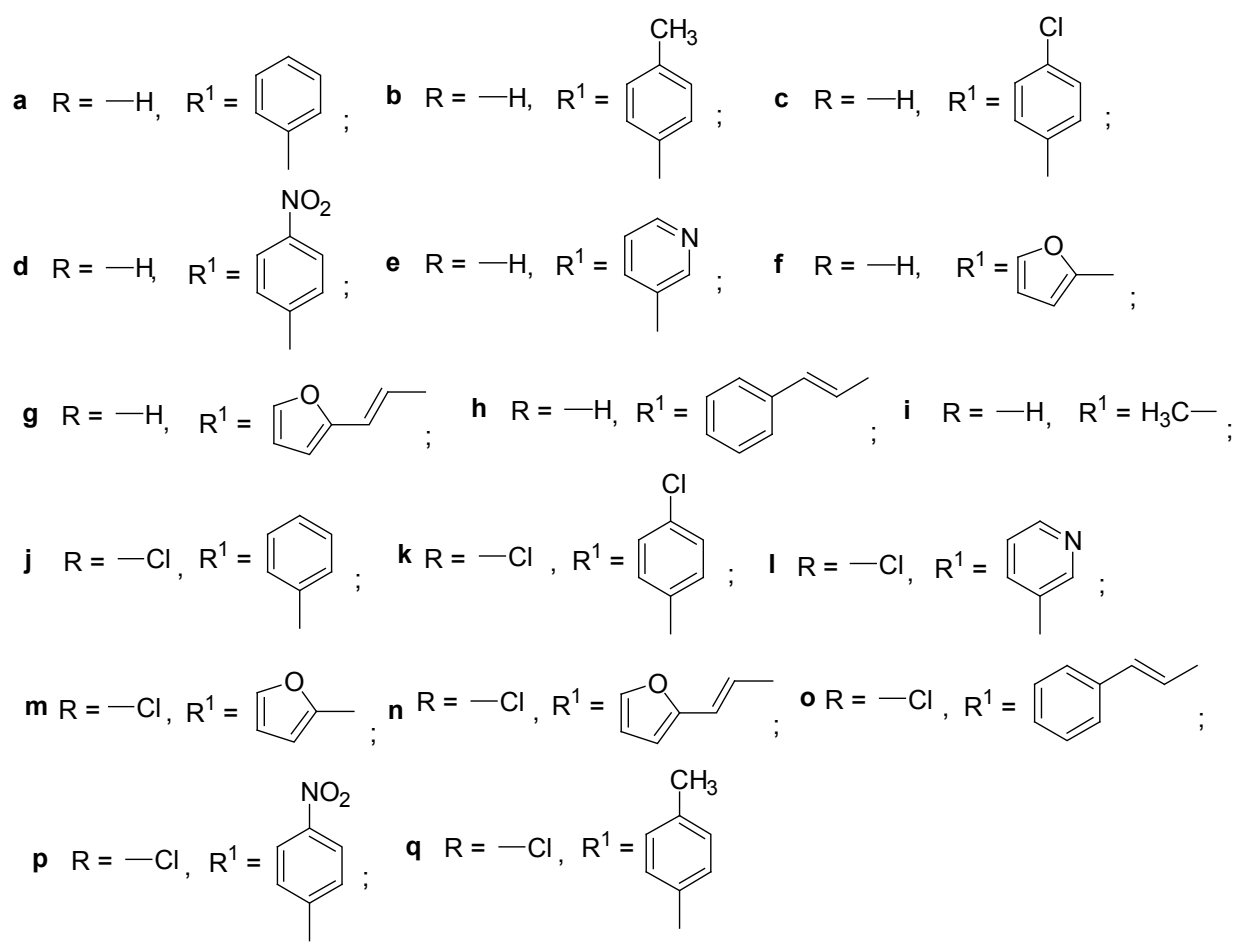

Scheme 1 
热, 反应结束时的温度 $130{ }^{\circ} \mathrm{C}$, TLC 跟踪反应终点. 当 反应完成后, 冷却至室温, 直接在 $200 \sim 300$ 目硅胶层析 柱中用石油醚和乙酸乙酯 $(V: V=3: 1)$ 对其进行分离 提纯, 经旋蒸、干燥后即得目的产物.

2,4,5-三苯基咪唑(4a): 无色针状晶体, 产率 50.7\%. m.p. $275 \sim 276{ }^{\circ} \mathrm{C} \quad$ (Lit. ${ }^{[22]} 277 \sim 278{ }^{\circ} \mathrm{C}$ ); ${ }^{1} \mathrm{H}$ NMR (DMSO- $\left.d_{6}, 300 \mathrm{MHz}\right) \delta: 12.69(\mathrm{~s}, 1 \mathrm{H}), 8.10$ (t, $J=1.7 \mathrm{~Hz}$, $1 \mathrm{H}), 8.08 \sim 8.06(\mathrm{~m}, 1 \mathrm{H}), 7.56(\mathrm{t}, J=1.8 \mathrm{~Hz}, 1 \mathrm{H}), 7.54(\mathrm{~d}$, $J=1.2 \mathrm{~Hz}, 1 \mathrm{H}), 7.53 \sim 7.47(\mathrm{~m}, 4 \mathrm{H}), 7.46 \sim 7.42(\mathrm{~m}, 2 \mathrm{H})$, 7.38 (ddd, $J=8.6,4.5,1.4 \mathrm{~Hz}, 2 \mathrm{H}), 7.34 \sim 7.27(\mathrm{~m}, 2 \mathrm{H})$, 7.23 (ddd, $J=7.2,3.7,1.4 \mathrm{~Hz}, 1 \mathrm{H}$ ); ${ }^{13} \mathrm{C}$ NMR (DMSO- $d_{6}$, $126 \mathrm{MHz}) \delta$ : 145.96, 137.58, 135.65, 131.56, 130.82, $129.13,128.93,128.69,128.64,128.23,127.53,126.96$, 125.65. HRMS calcd for $\mathrm{C}_{21} \mathrm{H}_{16} \mathrm{~N}_{2}[\mathrm{M}+\mathrm{H}]^{+}$297.1386, found 297.1387.

4,5-二苯基-2-(4-甲苯基)咪唑(4b): 乳白色针状晶 体, 产率 58.1\%. m.p. $230 \sim 231{ }^{\circ} \mathrm{C}$ (Lit. ${ }^{[22]} 230 \sim 232{ }^{\circ} \mathrm{C}$ ); ${ }^{1} \mathrm{H}$ NMR (DMSO- $\left.d_{6}, 500 \mathrm{MHz}\right) \delta: 12.60(\mathrm{~s}, 1 \mathrm{H}), 8.00$ (d, $J=7.5 \mathrm{~Hz}, 2 \mathrm{H}), 7.54(\mathrm{dd}, J=26.8,6.6 \mathrm{~Hz}, 4 \mathrm{H}), 7.43$ (d, $J=6.4 \mathrm{~Hz}, 2 \mathrm{H}), 7.37(\mathrm{~d}, J=6.4 \mathrm{~Hz}, 1 \mathrm{H}), 7.29$ (d, $J=7.5$ $\mathrm{Hz}, 4 \mathrm{H}), 7.23$ (d, $J=6.4 \mathrm{~Hz}, 1 \mathrm{H}), 2.35$ (s, 3H); ${ }^{13} \mathrm{C} \mathrm{NMR}$ (DMSO- $\left.d_{6}, 126 \mathrm{MHz}\right) \delta: 146.15,138.13,137.41,135.75$, $131.65,129.71,129.09,128.89,128.63,128.41,128.19$, $128.14,127.54,126.92,125.65,21.36$. HRMS calcd for $\mathrm{C}_{22} \mathrm{H}_{18} \mathrm{~N}_{2}[\mathrm{M}+\mathrm{H}]^{+} 311.1543$, found 311.1542 .

2-(4-氯基苯基)-4,5-二苯基咪唑(4c)：无色针状晶 体, 产率 66.6\%. m.p. $267 \sim 268{ }^{\circ} \mathrm{C}$ (Lit. ${ }^{[23]} 264 \sim 265{ }^{\circ} \mathrm{C}$ ); ${ }^{1} \mathrm{H}$ NMR (DMSO- $\left.d_{6}, 500 \mathrm{MHz}\right) \delta: 12.78(\mathrm{~s}, 1 \mathrm{H}), 8.10(\mathrm{~d}$, $J=8.6 \mathrm{~Hz}, 2 \mathrm{H}), 7.58 \sim 7.52(\mathrm{~m}, 4 \mathrm{H}), 7.50(\mathrm{~d}, J=7.4 \mathrm{~Hz}$, 2H), 7.45 (t, $J=7.5 \mathrm{~Hz}, 2 \mathrm{H}), 7.39$ (t, $J=7.2 \mathrm{~Hz}, 1 \mathrm{H}), 7.31$ $(\mathrm{t}, J=7.6 \mathrm{~Hz}, 2 \mathrm{H}), 7.23(\mathrm{t}, J=7.3 \mathrm{~Hz}, 1 \mathrm{H}) ;{ }^{13} \mathrm{C} \mathrm{NMR}$ (DMSO- $\left.d_{6}, 126 \mathrm{MHz}\right) \delta: 144.88,137.77,135.47,133.21$, $131.39,129.67,129.23,129.13,128.89,128.66,128.33$, 127.54, 127.30, 127.07. HRMS calcd for $\mathrm{C}_{21} \mathrm{H}_{15} \mathrm{ClN}_{2}[\mathrm{M}+$ $\mathrm{H}]^{+}$331.0997, found 331.0998.

2-(4-硝基苯基)-4,5-二苯基咪唑(4d)：黄色针状晶 体, 产率 76.5\%. m.p. $237 \sim 238{ }^{\circ} \mathrm{C}$ (Lit. ${ }^{[24]} 235 \sim 238{ }^{\circ} \mathrm{C}$ ); ${ }^{1} \mathrm{H}$ NMR (DMSO- $\left.d_{6}, 500 \mathrm{MHz}\right) \delta: 13.11(\mathrm{~s}, 1 \mathrm{H}), 8.35$ (d, $J=2.7 \mathrm{~Hz}, 4 \mathrm{H}), 7.55(\mathrm{~d}, J=7.4 \mathrm{~Hz}, 4 \mathrm{H}), 7.26 \sim 7.46(\mathrm{~m}$, $6 \mathrm{H}$ ); ${ }^{13} \mathrm{C}$ NMR (DMSO- $\left.d_{6}, 126 \mathrm{MHz}\right) \delta: 147.03,145.96$, $143.87,136.60,135.66,131.56,130.83,129.14,128.98$, $128.65,127.53,126.23,125.66,124.74$. HRMS calcd for $\mathrm{C}_{21} \mathrm{H}_{15} \mathrm{~N}_{3} \mathrm{O}_{2}[\mathrm{M}+\mathrm{H}]^{+}$342.1237, found 342.1239.

3-(4,5-二苯基咪唑基)吡啶 (4e): 乳白色针状晶体, 产率 60.6\%. m.p. $240 \sim 241{ }^{\circ} \mathrm{C} ;{ }^{1} \mathrm{H}$ NMR (DMSO- $d_{6}, 500$
MHz) $\delta$ : 12.90 (s, 1H), 9.26 (s, 1H), 8.57 (d, $J=4.7 \mathrm{~Hz}$, $1 \mathrm{H}), 8.40(\mathrm{~d}, J=8.0 \mathrm{~Hz}, 1 \mathrm{H}), 7.56(\mathrm{~d}, J=7.9 \mathrm{~Hz}, 2 \mathrm{H}), 7.52$ (d, $J=7.8 \mathrm{~Hz}, 3 \mathrm{H}), 7.46$ (t, $J=7.5 \mathrm{~Hz}, 2 \mathrm{H}), 7.40$ (t, $J=7.0$ $\mathrm{Hz}, 1 \mathrm{H}), 7.32$ (t, $J=7.6 \mathrm{~Hz}, 2 \mathrm{H}), 7.24$ (t, $J=7.3 \mathrm{~Hz}, 1 \mathrm{H})$; ${ }^{13} \mathrm{C}$ NMR (DMSO- $\left.d_{6}, 126 \mathrm{MHz}\right) \delta$ : 149.48, 146.90, $143.43,138.01,135.35,132.77,131.31,129.29,129.19$, $128.69,128.44,127.57,127.15,126.70,124.23$; IR (KBr) $v: 3030,2960,1600,1500,1450,1260,958,764,696,602$ $\mathrm{cm}^{-1}$. HRMS calcd for $\mathrm{C}_{20} \mathrm{H}_{15} \mathrm{~N}_{3}[\mathrm{M}+\mathrm{H}]^{+}$298.1339, found 298.1350 .

2-呋喃基-4,5-二苯基咪唑(4f): 灰色针状晶体, 产率 66.4\%. m.p. 202 $203{ }^{\circ} \mathrm{C}\left(\right.$ Lit. $\left.^{[22]} 200 \sim 201{ }^{\circ} \mathrm{C}\right) ;{ }^{1} \mathrm{H}$ NMR $\left(\mathrm{CDCl}_{3}, 300 \mathrm{MHz}\right) \delta: 7.53(\mathrm{~d}, J=7.0 \mathrm{~Hz}, 4 \mathrm{H}), 7.46(\mathrm{~s}$, $1 \mathrm{H}), 7.38 \sim 7.27(\mathrm{~m}, 6 \mathrm{H}), 7.02(\mathrm{~d}, J=3.3 \mathrm{~Hz}, 1 \mathrm{H}), 6.56 \sim$ $6.49(\mathrm{~m}, 1 \mathrm{H}) ;{ }^{13} \mathrm{C}$ NMR (DMSO- $\left.d_{6}, 126 \mathrm{MHz}\right) \delta$ : 146.16 , $143.53,139.05,137.46,135.43,131.27,129.13,128.81$, 128.67, 128.27, 127.99, 127.58, 127.08, 112.30, 107.91. HRMS calcd for $\mathrm{C}_{19} \mathrm{H}_{14} \mathrm{~N}_{2} \mathrm{O}[\mathrm{M}+\mathrm{H}]^{+}$287.1179, found 287.1174.

4,5-二苯基-2-(2-呋喃基乙烯基)咪唑(4g)：浅黄色针 状晶体, 产率 83.3\%. m.p. $194 \sim 196{ }^{\circ} \mathrm{C} ;{ }^{1} \mathrm{H}$ NMR $\left(\mathrm{CDCl}_{3}, 500 \mathrm{MHz}\right) \delta: 7.51(\mathrm{~d}, J=7.2 \mathrm{~Hz}, 4 \mathrm{H}), 7.39$ (d, $J=$ $1.1 \mathrm{~Hz}, 1 \mathrm{H}), 7.32(\mathrm{t}, J=7.3 \mathrm{~Hz}, 4 \mathrm{H}), 7.28(\mathrm{~d}, J=7.2 \mathrm{~Hz}$, $2 \mathrm{H}), 7.22(\mathrm{~d}, J=16.1 \mathrm{~Hz}, 1 \mathrm{H}), 6.85(\mathrm{~d}, J=16.1 \mathrm{~Hz}, 1 \mathrm{H})$, $6.41(\mathrm{dd}, J=3.2,1.8 \mathrm{~Hz}, 1 \mathrm{H}), 6.38(\mathrm{~d}, J=3.3 \mathrm{~Hz}, 1 \mathrm{H}) ;{ }^{13} \mathrm{C}$ NMR (DMSO- $\left.d_{6}, 126 \mathrm{MHz}\right) \delta: 152.29,145.16,142.80$, $132.50,129.15,128.56,127.84,127.47,119.36,113.69$, 111.87, 110.39; IR (KBr) v: 3060, 2960, 1600, 1580, 1480, 1450, 1320, 1260, 1200, 1070, 1010, 968, 883, 764, 696, $592 \mathrm{~cm}^{-1}$. HRMS calcd for $\mathrm{C}_{21} \mathrm{H}_{16} \mathrm{~N}_{2} \mathrm{O}[\mathrm{M}+\mathrm{H}]^{+}$ 313.1335, found 313.1334 .

4,5-二苯基-2-苯基乙烯基咪唑(4h): 黄色针状晶体, 产率 77.6\%. m.p. $227 \sim 229{ }^{\circ} \mathrm{C}$; ${ }^{1} \mathrm{H}$ NMR (DMSO- $d_{6}, 500$ MHz) $\delta: 12.60(\mathrm{~s}, 1 \mathrm{H}), 7.60$ (d, $J=7.4 \mathrm{~Hz}, 2 \mathrm{H}), 7.48$ (d, $J=22.8 \mathrm{~Hz}, 5 \mathrm{H}), 7.44 \sim 7.18(\mathrm{~m}, 9 \mathrm{H}), 7.10(\mathrm{~d}, J=16.5 \mathrm{~Hz}$, $1 \mathrm{H}) ;{ }^{13} \mathrm{C}$ NMR (DMSO- $\left.d_{6}, 126 \mathrm{MHz}\right) \delta: 145.71,136.91$, 130.45, 129.37, 128.92, 128.48, 128.03, 126.93, 117.68; IR (KBr) $v$ : 3030, 2960, 1600, 1500, 1450, 1200, 1070, 1030, $958,764,696,602 \mathrm{~cm}^{-1}$. HRMS calcd for $\mathrm{C}_{23} \mathrm{H}_{18} \mathrm{~N}_{2}[\mathrm{M}+$ $\mathrm{H}]^{+} 323.1543$, found 323.1543 .

2-甲基-4,5-二苯基咪唑(4i): 无色针状晶体, 产率 $38.5 \%$. m.p. $242 \sim 243{ }^{\circ} \mathrm{C}\left(\right.$ Lit. $\left.{ }^{[25]} 240 \sim 241{ }^{\circ} \mathrm{C}\right) ;{ }^{1} \mathrm{H}$ NMR (DMSO- $\left.d_{6}, 500 \mathrm{MHz}\right) \delta: 12.06(\mathrm{~s}, 1 \mathrm{H}), 7.45 \sim 7.18(\mathrm{~m}$, $10 \mathrm{H}), 2.34(\mathrm{~s}, 3 \mathrm{H}) ;{ }^{13} \mathrm{C}$ NMR (DMSO- $\left.d_{6}, 126 \mathrm{MHz}\right) \delta$ : $144.53,128.83,127.72,14.22$. HRMS calcd for $\mathrm{C}_{16} \mathrm{H}_{14} \mathrm{~N}_{2}$ 
$[\mathrm{M}+\mathrm{H}]^{+}$235.1230, found 235.1227.

4,5-二(4-氯基苯基)-2-苯基咪唑(4j): 乳白色针状晶 体, 产率 52.0\%. m.p. $291 \sim 293{ }^{\circ} \mathrm{C}$ (Lit. ${ }^{[23]} 290 \sim 291{ }^{\circ} \mathrm{C}$ ); ${ }^{1} \mathrm{H}$ NMR (DMSO- $\left.d_{6}, 500 \mathrm{MHz}\right) \delta: 12.79$ (s, 1H), 8.07 (d, $J=8.1 \mathrm{~Hz}, 2 \mathrm{H}), 7.57 \sim 7.51(\mathrm{~m}, 6 \mathrm{H}), 7.49$ (t, $J=7.9 \mathrm{~Hz}$, 2H), $7.40(\mathrm{dd}, J=7.2,5.1 \mathrm{~Hz}, 3 \mathrm{H}) ;{ }^{13} \mathrm{C}$ NMR (DMSO- $d_{6}$, $126 \mathrm{MHz}) \delta: 146.49,136.82,134.25,132.96,131.66$, $130.60,130.53,130.03,129.28,129.19,128.96,128.84$, 127.84, 125.75. HRMS calcd for $\mathrm{C}_{21} \mathrm{H}_{14} \mathrm{Cl}_{2} \mathrm{~N}_{2}[\mathrm{M}+\mathrm{H}]^{+}$ 365.0607 , found 365.0608 .

2,4,5-三(4-氯基苯基)咪唑(4k)：无色针状晶体，产 率 62.8\%. m.p. $261 \sim 262{ }^{\circ} \mathrm{C}$ (Lit. ${ }^{[25]} 263 \sim 264{ }^{\circ} \mathrm{C}$ ); ${ }^{1} \mathrm{H}$ NMR (DMSO- $\left.d_{6}, 500 \mathrm{MHz}\right) \delta: 12.87$ (s, 1H), 8.08 (d, $J=$ $8.5 \mathrm{~Hz}, 2 \mathrm{H}), 7.57 \sim 7.50$ (m, 8H), 7.39 (d, $J=8.4 \mathrm{~Hz}, 2 \mathrm{H})$; ${ }^{13} \mathrm{C}$ NMR (DMSO- $\left.d_{6}, 126 \mathrm{MHz}\right) \delta: 145.39,137.00$, 134.08, 133.48, 133.07, 131.77, 130.58, 129.87, 129.39, 129.30, 129.23, 128.87, 128.14, 127.41. HRMS calcd for $\mathrm{C}_{21} \mathrm{H}_{13} \mathrm{Cl}_{3} \mathrm{~N}_{2}[\mathrm{M}+\mathrm{H}]^{+}$399.0217, found 399.0226.

3-(4,5-二(4-氯基苯基)咪唑基)吡啶(41): 无色针状晶 体, 产率 61.6\%. m.p. $278 \sim 279{ }^{\circ} \mathrm{C} ;{ }^{1} \mathrm{H}$ NMR (DMSO- $d_{6}$, $500 \mathrm{MHz}) \delta: 13.00(\mathrm{~s}, 1 \mathrm{H}), 9.25(\mathrm{~s}, 1 \mathrm{H}), 8.58(\mathrm{~d}, J=4.1$ $\mathrm{Hz}, 1 \mathrm{H}), 8.38$ (d, J=7.7 Hz, 1H), 7.53 (s, 7H), 7.40 (s, $2 \mathrm{H}) ;{ }^{13} \mathrm{C}$ NMR (DMSO- $\left.d_{6}, 126 \mathrm{MHz}\right) \delta: 149.71,146.97$, $143.97,137.22$, 133.97, 133.18, 132.91, 131.87, 130.59, 129.82, 129.33, 128.90, 128.40, 126.46, 124.26; IR (KBr) $v: 3070,2990,1660,1600,1570,1480,1400,1330,1190$, 1090, 1010, 970, 831, 704, $604 \mathrm{~cm}^{-1}$. HRMS calcd for $\mathrm{C}_{20} \mathrm{H}_{13} \mathrm{Cl}_{2} \mathrm{~N}_{3}[\mathrm{M}+\mathrm{H}]^{+} 366.0559$, found 366.0563.

4,5-二(4-氯基苯基)-2-呋喃基咪唑(4m): 乳白色针 状晶体，产率 67.6\%. m.p. 269 $270{ }^{\circ} \mathrm{C}$; ${ }^{1} \mathrm{H} \mathrm{NMR}$ $\left(\mathrm{DMSO}-d_{6}, 500 \mathrm{MHz}\right) \delta: 12.94(\mathrm{~s}, 1 \mathrm{H}), 7.82$ (s, 1H), 7.50 (q, $J=8.4 \mathrm{~Hz}, 6 \mathrm{H}), 7.38(\mathrm{~d}, J=8.5 \mathrm{~Hz}, 2 \mathrm{H}), 6.98(\mathrm{~d}, J=$ $3.2 \mathrm{~Hz}, 1 \mathrm{H}), 6.66(\mathrm{dd}, J=3.0,1.6 \mathrm{~Hz}, 1 \mathrm{H}) ;{ }^{13} \mathrm{C} \mathrm{NMR}$ (DMSO- $\left.d_{6}, 126 \mathrm{MHz}\right) \delta: 145.88,143.74,139.48,136.73$, $134.03,132.99,131.77,130.53,129.76,129.28,128.87$, 127.14, 112.38, 108.27; IR (KBr) v: 3400, 3070, 2930, 1660, 1590, 1480, 1400, 1290, 1220, 1170, 1090, 1010, 970, 899, 835, 741, $592 \mathrm{~cm}^{-1}$. HRMS calcd for $\mathrm{C}_{19} \mathrm{H}_{12} \mathrm{Cl}_{2} \mathrm{~N}_{2} \mathrm{O}[\mathrm{M}+\mathrm{H}]^{+}$355.0402, found 355.0399.

4,5-二(4-氯基苯基)-2-(2-呋喃基乙烯基)咪唑(4n): 浅黄色针状晶体, 产率 $78.7 \%$. m.p. $230 \sim 231{ }^{\circ} \mathrm{C} ;{ }^{1} \mathrm{H}$ NMR (DMSO- $\left.d_{6}, 500 \mathrm{MHz}\right) \delta: 12.64(\mathrm{~s}, 1 \mathrm{H}), 7.73$ (d, $J=$ $1.5 \mathrm{~Hz}, 1 \mathrm{H}), 7.44$ (d, $J=52.0 \mathrm{~Hz}, 8 \mathrm{H}), 7.31$ (d, $J=16.3$ $\mathrm{Hz}, 1 \mathrm{H}), 6.81$ (d, $J=16.3 \mathrm{~Hz}, 1 \mathrm{H}), 6.67$ (d, $J=3.3 \mathrm{~Hz}$, $1 \mathrm{H}), 6.58(\mathrm{dd}, J=3.3,1.8 \mathrm{~Hz}, 1 \mathrm{H}) ;{ }^{13} \mathrm{C}$ NMR (DMSO- $d_{6}$,
$126 \mathrm{MHz}) \delta: 152.40,145.83,144.04,134.20,131.81$, $130.04,129.35,127.32,118.87,114.92,112.73,110.97$; IR (KBr) v: 3050, 2960, 1910, 1640, 1600, 1500, 1420, 1320, 1260, 1190, 1100, 1010, 970, 949, 833, 737, $592 \mathrm{~cm}^{-1}$. HRMS calcd for $\mathrm{C}_{21} \mathrm{H}_{14} \mathrm{Cl}_{2} \mathrm{~N}_{2} \mathrm{O}[\mathrm{M}+\mathrm{H}]^{+} 381.0557$, found 381.0556 .

4,5-二(4-氯基苯基)-2-苯基乙烯基咪唑(4o)：黄色针 状晶体，产率 $71.6 \%$. m.p. 228 $229{ }^{\circ} \mathrm{C}$; ${ }^{1} \mathrm{H}$ NMR $\left(\mathrm{CDCl}_{3}, 500 \mathrm{MHz}\right) \delta: 7.47(\mathrm{~d}, J=7.7 \mathrm{~Hz}, 2 \mathrm{H}), 7.41(\mathrm{~d}, J=$ $8.3 \mathrm{~Hz}, 5 \mathrm{H}), 7.34$ (t, $J=7.4 \mathrm{~Hz}, 2 \mathrm{H}), 7.30(\mathrm{~d}, J=8.3 \mathrm{~Hz}$, $5 \mathrm{H}), 7.01(\mathrm{~d}, J=16.5 \mathrm{~Hz}, 1 \mathrm{H}) ;{ }^{13} \mathrm{C} \mathrm{NMR}\left(\mathrm{CDCl}_{3}, 126\right.$ $\mathrm{MHz}) \delta: 145.80,135.86,133.64,132.50,130.42,129.08$, 128.92, 128.81, 128.63, 126.75, 115.38; IR (KBr) v: 3030, 2960, 1600, 1500, 1430, 1280, 1100, 1070, 970, 829, 754, $687,588 \mathrm{~cm}^{-1}$. HRMS calcd for $\mathrm{C}_{23} \mathrm{H}_{16} \mathrm{Cl}_{2} \mathrm{~N}_{2}[\mathrm{M}+\mathrm{H}]^{+}$ 391.0763, found 391.0757.

4,5-二(4-氯基苯基)-2-(4-硝基苯基)咪唑(4p): 金黄 色针状晶体，产率 $74.8 \%$. 276 $278{ }^{\circ} \mathrm{C}$; ${ }^{1} \mathrm{H} \mathrm{NMR}$ $\left(\mathrm{DMSO}-d_{6}, 500 \mathrm{MHz}\right) \delta$ : $13.23(\mathrm{~s}, 1 \mathrm{H}), 8.34$ (dd, $J=24.0$, $9.0 \mathrm{~Hz}, 4 \mathrm{H}), 7.55$ (s, 6H), 7.42 (d, J=7.5 Hz, 2H); ${ }^{13} \mathrm{C}$ NMR (DMSO- $\left.d_{6}, 126 \mathrm{MHz}\right) \delta: 147.22,144.36,138.09$, $136.30,133.73,133.45,132.10,130.72,129.60,129.51$, 129.39, 129.33, 128.97, 126.38, 124.77; IR (KBr) v: 3400, 2930, 1600, 1510, 1480, 1450, 1340, 1250, 1090, 1020, $966,833,758,710,696,673,604 \mathrm{~cm}^{-1}$. HRMS calcd for $\mathrm{C}_{21} \mathrm{H}_{13} \mathrm{Cl}_{2} \mathrm{~N}_{3} \mathrm{O}_{2}[\mathrm{M}+\mathrm{H}]^{+}$410.0458, found 410.0456.

4,5-二(4-氯基苯基)-2-(4-甲苯基)咪唑(4q)：无色针 状晶体, 产率 $60.5 \%$. m.p. $263 \sim 265{ }^{\circ} \mathrm{C}$ (Lit. ${ }^{[25]} 263 \sim 264$ $\left.{ }^{\circ} \mathrm{C}\right) ;{ }^{1} \mathrm{H}$ NMR (DMSO- $\left.d_{6}, 500 \mathrm{MHz}\right) \delta: 12.69$ (s, 1H), 7.96 $(\mathrm{d}, J=8.1 \mathrm{~Hz}, 2 \mathrm{H}), 7.53(\mathrm{~m}, 6 \mathrm{H}), 7.38(\mathrm{~d}, J=8.4 \mathrm{~Hz}, 2 \mathrm{H})$, $7.29(\mathrm{~d}, J=8.0 \mathrm{~Hz}, 2 \mathrm{H}), 2.35(\mathrm{~s}, 3 \mathrm{H}) ;{ }^{13} \mathrm{C}$ NMR $\left(\mathrm{DMSO}-d_{6}, 126 \mathrm{MHz}\right) \delta: 146.66,138.45,136.66,134.35$, $132.87,131.61,130.56,130.12,129.76,129.27,129.22$, 128.83, 127.88, 127.54, 125.73, 21.37. HRMS calcd for $\mathrm{C}_{22} \mathrm{H}_{16} \mathrm{Cl}_{2} \mathrm{~N}_{2}[\mathrm{M}+\mathrm{H}]^{+}$379.0762, found 379.0763 .

\section{2 结果与讨论}

在本研究中, 首先以苯偶姻衍生物和酰氯为原料在 温和的反应条件下合成了 17 种苯偶姻或取代苯偶姻单 酯化合物 3a 3q(其中 15 种未见报道)(Scheme 2). 实验 结果详见表 1 . 从表 1 可以看出, 合成单酯的收率在 $88.5 \%$ \% $6.2 \%$ 之间. 在探寻以苯偶姻单酯衍生物为原 料合成三取代咪唑时，选择苯甲酸-2-氧代-1,2-二苯基 乙基酯(2-oxo-1,2-diphenylethyl benzoate)和醋酸铵为原 料在传统加热、无溶剂条件下合成 2,4,5-三苯基-1-氢- 
咪唑, 虽然得到了目标产物, 但是所需的反应时间较长 $(2 \mathrm{~h})$, 收率较低. 另外, 在前人 ${ }^{[17]}$ 的研究中曾经以冰醋 酸为溶剂, 回流反应 $1 \mathrm{~h}$, 以几乎相等的收率 $(40 \%$ 左右) 获得目标产物和相应的噁唑. 孙继奎等 ${ }^{[19,20]}$ 在改变上述 合成条件的基础上, 以较高的收率合成了 2-取代-4,5-二 呋喃基噁唑衍生物. 在我们的研究中, 在不使用任何溶 剂的条件下, 将苯偶姻单酯或氯代苯偶姻单酯、醋酸铵 和三氧化二铝充分混合研磨后微波加热 5 或 $10 \mathrm{~min}$, 即 可以 $38.5 \% \sim 83.3 \%$ 的收率获得目标产物 $\mathbf{4 a \sim 4 q}$ (Eq. 1,
表 2). 但仅有极少量的上述报道 ${ }^{[19,20]}$ 的噁唑生成. 从表 2 可以看出, 当苯偶姻的脂肪酸酯与醋酸铵反应生成三 取代咪唑的 2-位取代基是甲基时, 收率最低(表 2, Entry 9). 在这里也尝试使用月桂酸苯偶姻酯为原料合成三取 代咪唑，但没有得到目标产物. 当咪唑的 2-位取代基是 带有共轭结构, 如苯环、呋喃环、吡啶环和苯环或呋喃 环上带有其它共轭基团时收率较高. 并且随着共轭结构 的增加(表 2, Entries 1，6 8 或 10,13 15), 收率升高. 这是因为苯偶姻单酯衍生物和醋酸铵受热分解产生氨

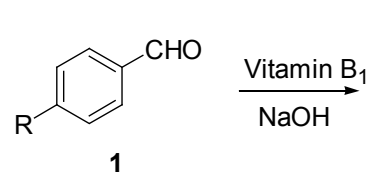<smiles>[R]c1ccc(C(=O)C(O)c2ccc([R])cc2)cc1</smiles>

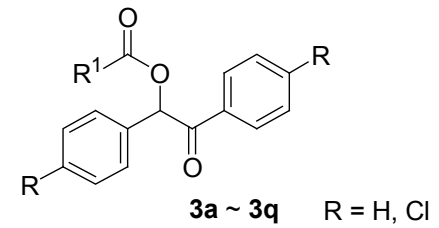

Scheme 2

表 1 苯偶姻单酯衍生物的合成

Table 1 Synthesis of benzoin carboxylates

Entry




\begin{tabular}{|c|c|c|c|c|}
\hline Entry & Acylating reagent & Product & Yield/\% & m.p. ${ }^{\circ} \mathrm{C}$ \\
\hline 7 & & $3 g$ & 96.2 & $99 \sim 101$ \\
\hline 8 & & $3 h$ & 95.9 & $97 \sim 99$ \\
\hline 9 & & $3 \mathbf{i}^{a}$ & 92.9 & $81 \sim 82$ \\
\hline 10 & & $3 \mathbf{j}$ & 93.1 & $104 \sim 105$ \\
\hline 11 & & $3 \mathrm{k}$ & 94.4 & $153 \sim 155$ \\
\hline 12 & & 31 & 91.7 & $115 \sim 117$ \\
\hline 13 & & $3 \mathrm{~m}$ & 88.5 & $202 \sim 204$ \\
\hline 14 & & $3 n$ & 94.8 & $187 \sim 189$ \\
\hline 15 & & 30 & 95.2 & $220 \sim 222$ \\
\hline 16 & & $3 p$ & 92.6 & $181 \sim 183$ \\
\hline 17 & & $3 q$ & 92.1 & $107 \sim 108$ \\
\hline
\end{tabular}

$\bar{a}$ 文献[18]中已报道的化合物. 
气的反应是亲核加成反应, 吸电子基的存在或共轭结构 的增加提高了羰基碳的亲电性和化合物的稳定性; 同 时, 在反应过程中醋酸铵受热分解产生的醋酸能与羰基 的氧原子形成氢键, 导致羰基碳的电子密度降低, 吸电 子能力增强, 这样使得带有一对孤对电子的氨气对羰基 碳的亲核进攻、酰基迁移以及在醋酸铵分解产生的醋酸 催化下的环合反应变得更容易进行, 并且长共轭结构的 存在提高了生成产物的稳定性, 因此产率高. 另外, 由
于微波可以降低反应活化能 ${ }^{[21]}$, 从而使反应速度加快, 缩短了反应时间.

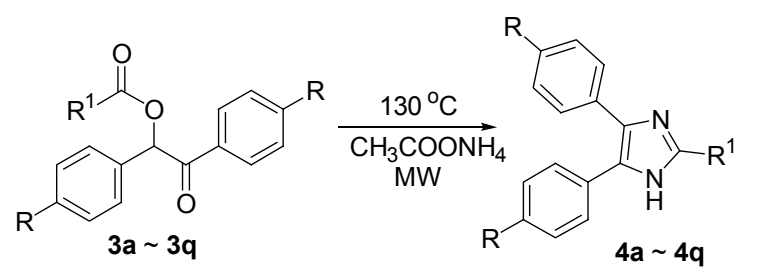

表 2 2-取代-4,5-二芳基咪唑的合成和产率

Table 2 Synthesis and yields of 2-substituted-4,5-diarylimidazoles

\begin{tabular}{|c|c|c|c|c|c|}
\hline Entry & $\mathrm{R}$ & $\mathrm{R}^{1}$ & Time/min & Product & Yield/\% \\
\hline 1 & $\mathrm{H}$ & & 5 & $4 a^{a}$ & 50.7 \\
\hline 2 & $\mathrm{H}$ & & 5 & $4 b^{a}$ & 58.1 \\
\hline 3 & $\mathrm{H}$ & & 5 & $4 c^{a}$ & 66.6 \\
\hline 4 & $\mathrm{H}$ & & 5 & $\mathbf{4 d}^{a}$ & 76.5 \\
\hline 5 & $\mathrm{H}$ & & 10 & $4 e^{a}$ & 60.6 \\
\hline 6 & $\mathrm{H}$ & & 5 & $4 f^{a}$ & 66.4 \\
\hline 7 & $\mathrm{H}$ & & 5 & $4 \mathrm{~g}$ & 83.3 \\
\hline 8 & $\mathrm{H}$ & & 5 & $4 h$ & 77.6 \\
\hline 9 & $\mathrm{H}$ & & 5 & $4 i^{a}$ & 38.5 \\
\hline 10 & $4-\mathrm{Cl}$ & & 5 & $4 j^{a}$ & 52.0 \\
\hline 11 & $4-\mathrm{Cl}$ & & 5 & $4 k^{a}$ & 62.8 \\
\hline 12 & $4-\mathrm{Cl}$ & & 10 & 41 & 61.6 \\
\hline
\end{tabular}




\begin{tabular}{|c|c|c|c|c|c|}
\hline Entry & $\mathrm{R}$ & $\mathrm{R}^{1}$ & Time/min & Product & Yield/\% \\
\hline 13 & $4-\mathrm{Cl}$ & & 5 & $4 m$ & 67.6 \\
\hline 14 & $4-\mathrm{Cl}$ & & 5 & $4 n$ & 78.7 \\
\hline 15 & $4-\mathrm{Cl}$ & & 5 & 40 & 71.6 \\
\hline 16 & $4-\mathrm{Cl}$ & & 5 & $4 p$ & 70.8 \\
\hline 17 & $4-\mathrm{Cl}$ & & 5 & $\mathbf{4} \mathbf{q}^{a}$ & 60.5 \\
\hline
\end{tabular}

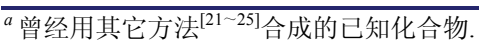

\section{3 结论}

本文揭示了一种在微波促进下, 以苯偶姻单酯或氯 代苯偶姻单酯为原料, 在无溶剂条件下以三氧化铝为固 载体与醋酸铵作用合成三取代咪唑衍生物的新方法. 这种方法与传统方法相比, 具有反应时间短、产率较高、 操作简单、不需使用溶剂, 因此其是一种操作简单、能 耗低的节能环保的合成方法. 这为含有咪唑环类化合物 的合成提供一种便捷的途径. 另外, 本研究组关于呋喃 取代咪唑化合物的应用研究已取得了一定的研究成果, 有关其它相关化合物的合成与应用研究在本实验室正 在进行中.

\section{References}

[1] (a) Lombardino, J. G.; Wiseman, E. H. J. Med. Chem. 1974, 17, 1182 .

(b) Frazen, R. G. J. Comb. Chem. 2000, 2, 195

(c) Fantini, M.; Zuliani, V.; Spotti, M. A.; Rivara, M. J. Comb. Chem. 2010, 12, 181.

(d) Soh, C. H; Lam, Y. J. Comb. Chem. 2010, 12, 286.

(e) Liebl, R.; Handte, R.; Mildenberger, H.; Bauer, K.; Bieringer, H. DE 3604042 [Chem. Abstr. 1988, 108, 6018].

[2] (a) Kenichiro, N.; Hiromi, Y.; Naotaka, K. Anal. Chim. Acta 1995, 303(1), 103

(b) Sun, Y.; Nakashima, M. N.; Takahashi, M.; Kuroda, N.; Nakashima, K. Biomed. Chromatogr. 2002, 16, 319.

(c) Kang, J.; Zhang, Y.-M.; Han, L.; Tang, J.-L.; Wang, S.-J.; Zhang, Y.-H. J. Photochem. Photobiol. A 2011, 217, 376.

(d) Kang, J; Zhang, Y.-M.; Huang, Z.-X.; Han, L; Tang, J.-L.; Wang, S.-J.; Zhang, Y.-H. J. Fluoresc. 2011, 21, 1607.

[3] Liu, S.-F.; Wu, Q.-G.; Schmider, H. L.; Aziz, H.; Hu, N.-X.; Popović, Z.; Wang, S.-N. J. Am. Chem. Soc. 2000, 122, 3671.

[4] Cai, Y.-P.; Chen, C.-L.; Su, C.-Y.; Kang, B.-S. Inorg. Chim. Acta
2003, 342, 107.

[5] Schuster, M. F. H.; Meyer, W. H.; Schuster, M.; Kreuer, K. D. Chem. Mater. 2004, 16, 329

[6] Zhao, L.; Li, S. B.; Wen, G. A.; Peng, B.; Huang, W. Mater. Chem. Phys. 2006, 100, 460.

[7] Horton, D. A.; Bourne, G. T.; Smyth, M. L. Chem. Rev. 2003, 103, 893.

[8] Nefzi, A.; Ostresh, J. M.; Houghten, R. A. Chem. Rev. 1997, 97, 449

[9] Fang, Z.-J.; Wang, S.-M.; Zhao, L.; Xu, Z.; Ren, J.; Wang, X.-B.; Yang, Q.-F. Mater. Lett. 2007, 61, 4803.

[10] (a) Ma, W.-Z.; Liu, S.-W.; Hu, J. Chem. Reag. 1997, 19, 281 (in Chinese).

(马文展，刘少文，胡建，化学试剂, 1997, 19, 281.)

(b) Jiang, Z.-L.; Yan, J.-M.; Xie, R.-G. Chin. J. Synth. Chem. 1998 6, 11 (in Chinese)

(蒋宗林, 鄢家明, 谢如刚, 合成化学, 1998, 6, 11.)

(c) Lipshutz, B. H.; Morey, M. C. J. Org. Chem. 1983, 48, 3745.

(d) Siddiqui, S. A.; Narkhede, U. C.; Palimkar, S. S.; Daniel, T.; Lahoti, R. J.; Srinivasan, K. V. Tetrahedron 2005, 61, 3539.

(e) Balalaie, S.; Hashemi, M. M.; Akhbari, M. Tetrahedron Lett. 2003, 44, 1709.

(f) Wolkenberg, S. E.; Wisnoski, D. D.; Leister, W. H.; Wang, Y.; Zhao, Z.; Lindsley, W. Org. Lett. 2004, 6, 1453.

(g) Wang, S.-J.; Gu, Q.; Chen, X.-D.; Zhao, T.-Q.; Zhang, Y.-M. Eur. J. Chem. 2011, 2(2), 173.

(h) Zhang, H.-L.; Li, H.-G.; Wu, H.-H. Chin. J. Org. Chem. 2011, 31(9), 1433 (in Chinese).

(张焕, 李海岗, 吴海虹, 有机化学, 2011, 31(9), 1433.)

[11] (a) Siddiqui, S. A.; Narkhede, U. C.; Palimkar, S. S.; Daniel, T.; Lahoti, R. J.; Srinivasan, K. V. Tetrahedron 2005, 61, 3539.

(b) Karimi-Jaberi, Z.; Barekat, M. Chin. Chem. Lett. 2010, 21, 1183.

(c) Balalaie, S.; Hashemi, M. M.; Akhbari, M. Tetrahedron Lett. 2003, 44, 1709.

(d) Wolkenberg, S. E.; Wisnoski, D. D.; Leister, W. H.; Wang, Y.; Zhao, Z.; Lindsley, C. W. Org. Lett. 2004, 6, 1453.

(e) Niknam, K.; Mohammadizadeh, M. R.; Mirzaee, S.; Saberi, D. 
Chin. J. Chem. 2010, 28, 663.

(f) Shelke, K. F.; Kakade, G. K.; Shingate, B.; Shingare, M. Rasayan J. Chem. 2008, 1, 489.

(g) Das, B.; Srinivas, Y; Holla, H.; Krishnaiah, M.; Narender, R. Chem. Lett. 2007, 36, 1270.

(h) Bhosale, S. V.; Mohan B. K.; Nalage, S. V.; Bhosale, D. S.; Pandhare, S. L.; Kotbagi, T. V.; Umbarkar, S. B.; Dongare, M. K. Synth. Commun. 2011, 41, 762.

[12] Alexander, U; Yuri, L.-K. Tetrahedron Lett. 2000, 41, 5031.

[13] Balalaie, S; Arabanian, A. Green Chem. 2000, 2, 274.

[14] (a) Jin, J.; Zhang, J.; Liu, F.; Shang, W.; Xin, Y.; Zhu, S. Chin. J. Chem. 2010, 28, 1217.

(b) Bai, S.; Song, B.; Bhadury, P. S.; Yang, S.; Hu, D.; Xue, W. Chin. J. Chem. 2011, 29, 109.

(c) Jin, J.; Zhang, J.; Shang, W.; Zhu, S. Chin. J. Chem. 2011, 29, 2643.

(d) Zhang, X.; Luo, K.; Chen, W.; Wang, L. Chin. J. Chem. 2011, 29, 2209.

(e) Niu, H.-Y.; Yuan, T.-F.; Qu, G.-R.; Li, D.-Y.; Mao, R.-Z.; Jin, X.; Yang, X.-N.; Guo, H.-M. Chin. J. Org. Chem. 2011, 31, 1613 (in Chinese).

(牛红英, 袁腾飞, 渠桂荣, 李德阳, 毛润泽, 金金金, 杨西宁, 郭 海明, 有机化学, 2011, 31, 1613.)

[15] Gao, D.-W.; Yu, H.-F.; Jia, J.-L.; Hua, S.-Y.; Chen, X.-D. Acta Sci. Nat. Univ. Jilinensis 1998, (1), 107 (in Chinese).

(高大维, 于化锋, 贾晋礼, 华士英, 陈晓东, 吉林大学自然科学 学报, 1998, (1), 107.)
[16] Wang, Z.-J.; Jiang, Y.-C. Acta Zhengzhou Grain College 2000, 21(2), 28 (in Chinese). (王志军, 姜延程, 郑州粮食学院学报, 2000, 21(2), 28.)

[17] The Teaching and Research Group of Organic Chemistry in Chemical Department of Lanzhou and Fudan Universities Experiment of the Organic Chemistry, 2nd ed., Higher Education Press, Beijing, 1994, pp. 206 208 (in Chinese). (兰州大学、复旦大学化学系有机教研组编, 有机化学实验(第二 版), 高等教育出版社, 北京, 1994, pp. 207〜208.)

[18] Strzbny, P. P. E.; Van Es, T.; Backeberg, O. G. J. Org. Chem. 1963, $28,3381$.

[19] Pei, W.-W.; Li, S.-H.; Nie, X.-P.; Li, Y.-W.; Pei, J; Wu, B.-Z.; Chen, J.; Ye, X.-L. Synthesis 1998, 1298.

[20] Sun, J.-K.; Zhang, F.; Gao, D.-W.; Zhang, Y.-M.; Zhao, T.-Q.; Chen, X.-D. Chin. J. Org. Chem. 2008, 28, 1611 (in Chinese). (孙继奎, 张峰, 李亚卓, 高大维, 张玉敏, 赵天琦, 陈晓东, 有 机化学, 2008, 28, 1611.)

[21] Langa, F.; de la Cruz, P.; de la Hoz, A.; Diaz-Ortiz, A.; Diez-Barra, E. Contemp. Org. Synth. 1997, 4, 373.

[22] Joshi, R. S.; Mandhane, P. G.; Shaikh, M. U.; Kale, R. P.; Gill, C. H. Chin. Chem. Lett. 2010, 21, 429.

[23] Zhou, J.-F.; Gong, G.-X.; Sun, X.-J. Synth. Commun. 2010, 40, 1134.

[24] Oskooie, H. A.; Alimohammadi, Z.; Heravi, M. M. Heteroat. Chem. 2006, 17, 699.

[25] Samai, S.; Nandi, G. C.; Singh, P.; Singh, M. S. Tetrahedron 2009, $65,10155$. 